\title{
Walter Krumholz - ein Leben für die Dokumentation
}

https://doi.org/10.1515/iwp-2019-2013

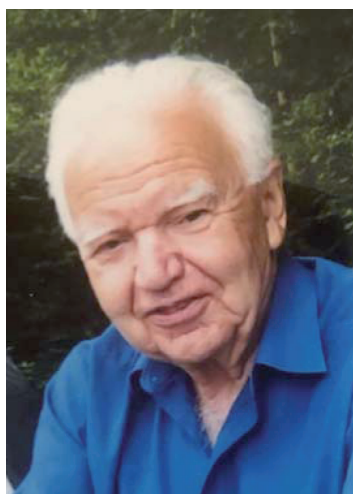

Rolf-Walter Krumholz (geb. am 6. April 1924 in Hannover) ist am 10. März 2019 in Berlin gestorben. Sein Berufsweg hatte ihn schon früh mit der Dokumentation in Berührung gebracht.

Bereits während seines Studiums der Politikwissenschaft an der Deutschen Hochschule für Politik DHfP in Berlin wurde er 1949 Assistent des Direktors, Otto Suhr. Nach seiner Diplomprüfung übernahm er 1952 die Leitung des Archivs, später der Dokumentation der DHfP (nach 1958 des Otto-Suhr-Instituts der FU Berlin) und wurde 1965 schließlich zum wissenschaftlichen Geschäftsführer der damals neu gegründeten „Leitstelle Politische Dokumentation“ (LPD) an der FU Berlin bestellt. Die Leitstelle sollte die Zusammenarbeit der Informations- und Dokumentationsstellen im Bereich der Politik sicherstellen und die Benutzung politischer Dokumente erleichtern. Dazu dienten die beiden von Krumholz herausgegebenen Referatedienste „Politische Dokumentation (poldok)“, für den von 1965 bis 1988 regelmäßig 200 deutschsprachigen Zeitschriften ausgewertet wurden, und „Politikwissenschaftliche Forschung“, der von 1967 bis 1977 eine Übersicht über Forschungsarbeiten in Deutschland, Österreich und der Schweiz bot. poldok wurde seit 1977 als Datenbank auf dem Host Genios-Wirtschaftsdatenbanken angeboten.

1967 wurde Krumholz an der Technischen Hochschule in Braunschweig mit einer Arbeit über „Die politische Dokumentation in der Bundesrepublik Deutschland“ zum Dr. phil. promoviert. Es folgte 1974 die Ernennung zum Honorarprofessor für Politische Wissenschaften an der FU Ber- lin, wo Krumholz am Otto-Suhr-Institut u. a. Informationspolitik lehrte.

Walter Krumholz war 1962 als Mitglied in die Deutsche Gesellschaft für Dokumentation (DGD) eingetreten. Bei der Mitgliederversammlung in Darmstadt wurde er 1969 zum Schriftführer gewählt, drei Jahre später in Bad Dürkheim dann zum Schatzmeister. Als Schatzmeister der DGD erlebte er bis 1984 drei Präsidentschaften von Lutterbeck über Möhring zu Canisius. Es waren Zeiten des finanziellen Wachstums. Heinz Lechmann setzte sich im Bundesministerium für Forschung und Technologie für die Grundlagenforschung und der Pflege internationaler Beziehungen im Bereich der Dokumentation ein. So nahm Krumholz sich im Präsidium der DGD nach 1972 vor allem der internationalen Aufgaben an, was nicht zuletzt das vom BMFT finanzierte Großprojekt „Internationale Aufgaben der DGD" einschloss. Sein Aufgabengebiet schloss reichhaltige Kontakte ins Ausland, oft verbunden mit Reisen oder dem Empfang internationaler Gäste in Deutschland, ein. Besonders in Erinnerung bleibt die Mitbegründung von WERTID (Western European Round Table on Information and Documentation), der ab 1994 als Rat der Informationsgesellschaften (ECIA) firmierte und u. a. die Europäische Zertifizierung für Informationsfachleute in Europa auf den Weg gebracht hat. Krumholz war bis zu ihrer Auflösung als honorary fellow der DGI aktiv. Wie Wolfrudolf Laux in seiner Gratulation zum 80 Geburtstag feststellte, war Krumholz nicht nur der „Finanzminister“, sondern auch der „Außenminister“ der DGD. Seine Reisefreude war sprichwörtlich.

Für die Friedrich-Naumann-Stiftung erarbeitete er ein Curriculum für Dokumentationskurse, an denen er viele Jahre als Dozent in Tunesien (Tunis), Uganda (Kampala) und Marokko (Rhabat) teilnahm.

Auch in der Normungsarbeit hinterließ Walter Krumholz seine Spuren. Er arbeitete in den Ausschüssen für Datenerfassungselemente des DIN und der ISO mit, übernahm im DIN den Vorsitz der Normenausschüsse für Klassifikation und für Länderzeichen und war Chairman der UDC-Assembly der FID, Den Haag.

Nach der Jahrtausendwende übersiedelte Krumholz für einige Jahre ins badische Wehr und begann sich dort wissenschaftlich und in Gesprächskreisen mit der Hirnforschung und dem Thema „Der Mensch (das biologische Wesen), das von der Politik und Politischen Wissenschaft vergessene Objekt“ auseinander zu setzen. Sein letzter 
Beitrag in der IWP „Grenzen der physiologischen Informationsverarbeitung des Menschen“ erschien in Band 55 (2004) Heft 5, S. 293-297 und warnte vor dem „Informationstrommelfeuer“, dem sich die Menschen durch die neuen Kommunikationsmedien aussetzen sowie der damit einhergehenden Gefahr, durch die absichtliche Ausnutzung der physiologischen Grenzen des menschlichen Informationsverarbeitungsvermögens „drohen die Menschen zur manipulierbaren Masse zu verkommen“.

Walter Krumholz verstarb in seiner Wahlheimat Berlin nach einem langen, ereignisreichen und erfüllten Leben. Die DGI wird das Andenken an ihn bewahren.

Marlies Ockenfeld, Darmstadt

\section{Prof. Dr. Walther Umstätter †}

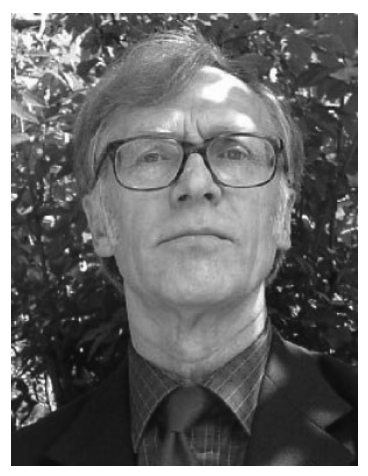

Walther Umstätter starb am 6. März 2019 nach schwerer Krankheit. Er gehörte zu den leider wenigen Grenzgängern zwischen Bibliothek und Dokumentation, wie man die Informationserschließung und -vermittlung in jenen Jahren nannte, als Umstätter seine berufliche Laufbahn begann. Entsprechend hat er sich die ungute Trennung von Bibliothekswissenschaft und Informationswissenschaft nie $\mathrm{zu}$ eigen gemacht.

Umstätter wurde am 12. Juni 1941 in Rumänien geboren; seine Eltern gehörten zur deutschsprachigen Minderheit in der Industriestadt Ploiești. Er studierte an der Freien Universität Berlin und wurde dort 1978 im Fach Biologie promoviert. Als einer der ersten Onliner in Deutschland baute er ab 1975 an der Universitätsbibliothek Ulm die erste Online-Informationsvermittlungsstelle an einer deutschen Universitätsbibliothek mit Anschluss an das damalige DIMDINET auf. Mit seiner Kompetenz als promovierter Biologe unterstützte er die Hochschulangehörigen und externe Wissenschaftler, insbesondere die Mediziner, indem er Online-Recherchen in den Biologie- und

\section{Literatur}

Arntz, Helmut: Walter Krumholz 65. In: Nachrichten für Dokumentation 40 (1989), S. 259.

Krumholz, Walter: Grenzen der physiologischen Informationsverarbeitung des Menschen. In: IWP 55 (2004) 5, S. 293-297.

Laux, Wolfrudolf: Walter Krumholz 80 Jahre plus. In: IWP 56 (2005) Nr. 3, S. 200.

Eintrag „Krumholz, Walter“ in Munzinger Online/Personen - Internationales Biographisches Archiv, http://www.munzinger.de/doc ument/00000014604.

Medizindatenbanken durchführte. Daneben unterrichtete er Information Retrieval an der Schule für Medizinische Dokumentationsassistenten in Ulm. Seine Erfahrung gab er gerne und produktiv weiter und half so mit, dass DIMDI die Nutzerfreundlichkeit der Datenbankrecherchen mit einer neuen Retrievalsprache verbessern konnte. Bei den Online-Tagungen der DGD (heute DGI) hielt er regelmäßig praxisorientierte Vorträge.

1982 wurde Umstätter zum Professor an die Fachhochschule für Bibliotheks- und Dokumentationswesen Köln (FHBD), heute ein Fachbereich der Technischen Hochschule Köln, berufen und lehrte dort bis 1994 Online-Retrieval, Fachbibliografie und Dokumentation. Die dortige gemeinsame Ausbildung für den Bibliotheks- und Dokumentationsbereich (Diplom) entsprach seinem Verständnis von Informationsvermittlung. In der Lehre betonte er die Rolle der Informationstechnologien, was sich u.a. in den Themen der damaligen Abschlussarbeiten ausdrückte, wie etwa Möglichkeiten des Einsatzes von PCs in Universitätsbibliotheken.

An der Universität Köln war er Lehrbeauftragter im damals noch bestehenden Studiengang Bibliothekswissenschaft. 1994 erhielt Umstätter den Ruf an die Humboldt-Universität zu Berlin, wo es darum ging, das seit 1955 bestehende Institut für Bibliothekswissenschaft zu erneuern. Die von Umstätter angestrebte Erneuerung fand enge Grenzen, weil eine nennenswerte Anzahl von Neuberufungen erst nach seiner Emeritierung 2006 erfolgen konnte, nachdem das Institut zunächst von ersatzloser Streichung bedroht war und dann dank einer erneuten Neuprofilierung gesicherte Ressourcen erkämpfen konnte.

Umstätter schrieb eine Reihe von Lehrbüchern, zuerst 1981 die Einführung in die Literaturdokumentation und Informationsvermittlung (Saur) zusammen mit Margarete 
Rehm, die damals Direktorin der Universitätsbibliothek Ulm war. Es folgte 1997 das Lehrbuch der Bibliotheksverwaltung (zs. mit seiner Kollegin Gisela Ewert, Hiersemann); es erschien 2011 in 2. Auflage unter dem Titel Lehrbuch des Bibliotheksmanagements. Der Titel der ersten Auflage war der Wunsch des Verlags, der mit dieser Titelformulierung an seinen gleichnamigen Titel aus den 1950er Jahren anknüpfen wollte. 2005 erschien die Einführung in die Katalogkunde (zs. mit Roland Wagner-Döbler, Hiersemann); der Untertitel Vom Zettelkasten zur Suchmaschine zeigt die Richtung an, die dieses Lehrbuch weist.

Seine insgesamt über 150 Publikationen, darunter etliche Monografien und Kongressschriften, dokumentieren ein sehr breites Interesse an allem, was mit Information und ihrer Bedeutung für die Gesellschaft zusammenhängt; das Spektrum der Themen, die Umstätter behandelte, reicht von Online-Literaturvermittlung bis zur Wissensorganisation, von digitalen Bibliotheken bis zum Selbstverständnis des Faches.

Ganz besonders wichtig waren ihm stets eine präzise Ausdrucksweise und klare Begriffsbestimmungen. Umstätters Semiotischer Thesaurus ist vordergründing eine Sammlung von Definitionen der Termini der Bibliotheksund Informationswissenschaft. Der Thesaurus sollte jedoch auch als Basis der Zusammenarbeit von Autoren und Autorinnen verschiedener Disziplinen dienen, die darauf aufbauend ein gemeinsames Begriffsverständnis entwickeln sollten. Auch für Evaluationen forderte Umstätter zuverlässige Kriterien.

Aus Anlass seines 65. Geburtstags widmete ihm sein Institut eine Festschrift (Vom Wandel der Wissensorganisation im Informationszeitalter. Hrsg. von P. Hauke u. K. Umlauf. Bad Honnef: Bock+Herchen 2006), die gleichzeitig als gedrucktes Buch und alsE-Booknach OAI-Standards erschien.
Über Forschung und Lehre hinaus engagierte sich Umstätter vielfältig innerhalb und außerhalb der Universität. Er war viele Jahre lang Geschäftsführender Direktor des Instituts für Bibliothekswissenschaft an der HumboldtUniversität, dessen Umbenennung in Institut für Bibliotheks- und Informationswissenschaft kurz vor seiner Emeritierung erfolgte, ferner etliche Jahre lang Mitglied im Fakultätsrat. Zu seinen Engagements außerhalb der Universität gehörten seine Vizepräsidentschaft der Gesellschaft für Wissenschaftsforschung, die Tagungen zur Szientometrie und Wissenschaftssoziologie durchführt, die Mitgliedschaften im Herausgeberbeirat der Nachrichten für Dokumentation (heute IWP)), in den Vorständen der $D G D$, in die er 1983 eintrat, des Deutschen Bibliotheksverbands und der deutschen Sektion der International Society for Knowledge Organization, ferner in zahlreichen Fachgremien und -beiräten, schließlich im Förderverein der Öffentlichen Bibliothek seines Wohnortes Altlandsberg bei Berlin.

Nach seiner Emeritierung befasste er sich weiterhin vor allem mit Problemen der Informationstheorie, der Szientometrie und der Frage, wie sich Wissen und Information messen lassen. Die wissenschaftliche Fundierung und Fokussierung der Informationswissenschaft war ihm ein großes Anliegen. Er schrieb Rezensionen und beteiligte sich an fachlichen Diskussionen, etwa in inetbib.

Walther Umstätter war ein scharfsinniger und Präzision verlangender Doktorvater zahlreicher Promovenden, darunter Kandidaten aus China und dem Iran. Wer persönlich mit ihm zu tun hatte, schätzte seine unprätentiöse Art, seine naturwissenschaftlich geprägte sachbezogene Argumentationsweise und seine nüchterne Offenheit. Die vielfältigen Anregungen und Denkanstöße in seinen $\mathrm{Pu}$ blikationen sind weiterhin lesenswert und bilden ein bleibendes Erbe für unsere Profession.

Konrad Umlauf, Niederstetten und Marlies Ockenfeld, Darmstadt

teraturrecherche für die Chemie- und Life Science-Industrie sowie in der Beschaffung von Originalliteratur entstand so ein Service-Unternehmen, das sowohl die aus der Hoechst AG hervorgegangenen Nachfolgefirmen als auch andere Firmen im Industriepark Höchst mit Informationen und Dokumenten versorgte sowie Systemlösungen in den Bereichen Application Services, Internet Services, Datenbank Hosting und Netzwerk-Management entwickelte, die auf die spezifischen Bedürfnisse der Kunden angepasst sind. Dr. Jürgen Sander war seit 2006 im Ruhestand, blieb aber der Literatur- und Patentrecherche weiter verbunden. 